\title{
Impacto das tecnologias inovadoras na vida de diabéticos adultos: revisão integrativa
}

\author{
Impact of innovative technologies on the lives of adult diabetics: an integrative review \\ Impacto de las tecnologías innovadoras en la vida de los diabéticos adultos: revisión integrativa
}

\author{
Rodrigo Domingos de Souza'; Marcos Antonio Ferreira Júniorl'; Leandro Hisao Modesto Ikedall'; \\ Marisa Dias Rolan Loureiro'v; Maria Gorette dos Reis'; Oleci Pereira Frotalv
}

\begin{abstract}
RESUMO
Objetivo: revisar artigos de pesquisa visando a análise do impacto de tecnologias inovadoras na vida de diabéticos adultos. Método: trata-se de uma revisão integrativa de produção levantada nas bases de dados MEDLINE, CINAHL, LILACS, Web of Science, Springer Link e bibliotecas eletrônicas SciELO e PubMed. Foram incluídos ensaios clínicos randomizados, em Português, Inglês ou Espanhol, entre 2012 e 2016. Resultados: Identificaram-se 669 publicações, das quais 12 compuseram a amostra. Identificaram-se 11 tecnologias distintas, que envolveram desde a utilização de aplicativos para smartphones, farmacoterapias, dispositivos de monitorização até intervenções especializadas. A maioria impactou positivamente na vida dos diabéticos para o controle metabólico eficaz e dos níveis de Hemoglobina Glicada, redução de episódios de hiper/hipoglicemia, maior adesão ao tratamento, entre outros, enquanto apenas uma apresentou aspectos desfavoráveis. Conclusão: há a necessidade de aprimoramento das tecnologias, bem como realização de estudos com desenhos de pesquisa de maior acurácia e com métodos mais robustos de investigação.
\end{abstract}

Descritores: Inovação; terapêutica; diabetes mellitus; qualidade de vida.

\begin{abstract}
Objective: to review research articles with a view to examining the impact of innovative technologies on the lives of adult diabetics. Method: this integrative review of production found in the MEDLINE, CINAHL, LILACS, Web of Science, Springer Link and SciELO and PubMed electronic libraries included randomized clinical trials in Portuguese, English or Spanish published between 2012 and 2016. Results: of the 669 publications identified, 12 formed the sample, in which 11 different technologies were identified, ranging from the use of smartphone applications, pharmacotherapies, and monitoring devices to specialized interventions. Most impacted the lives of diabetics beneficially towards effectively controlling metabolic and glycated hemoglobin levels, reducing episodes of hyperor hypoglycemia, increasing treatment adherence, and so on, while only one displayed adverse effects. Conclusion: there is a need for improved technologies, as well as for studies with more accurate research designs and more robust research methods.

Descriptors: Innovation; therapeutics; diabetes mellitus; quality of life.
\end{abstract}

\section{RESUMEN}

Objetivo: revisar artículos de investigación con vistas a analizar el impacto de las tecnologías innovadoras en la vida de diabéticos adultos. Método: se trata de una revisión integrativa recopilada de las bases de datos MEDLINE, CINAHL, LILACS, Web of Science, Springer Link y de las bibliotecas electrónicas SciELO y PubMed. Se incluyeron los ensayos clínicos randomizados en portugués, inglés o español, entre 2012 y 2016. Resultados: se identificaron 669 publicaciones, de las cuales 12 compusieron la muestra. Se identificaron 11 tecnologías distintas que involucraron desde el uso de aplicaciones para smartphones, farmacoterapias, dispositivos de monitorización hasta intervenciones especializadas. La mayoría tuvo impacto positivo en la vida de los diabéticos para el control metabólico eficaz y de los niveles de Hemoglobina Glicolisada, reducción de episodios de hiper / hipoglucemia, mayor adhesión al tratamiento, entre otros; sólo una presentó aspectos desfavorables. Conclusión: es necesario el perfeccionamiento de las tecnologías, así como realización de estudios con dibujos de investigación de mayor precisión y con métodos más robustos de investigación.

Descriptores: Innovación; terapéutica; diabetes mellitus; calidad de vida.

\section{INTRODUÇÃO}

As doenças crônicas não transmissíveis (DCNT) têm apresentado proporções epidêmicas em todo o mundo. No Brasil, correspondem a $66 \%$ da carga total de doenças. O Diabetes Mellitus (DM) é umas das doenças mais prevalentes entre as DCNT. Afeta indivíduos de todas as idades, etnias, sexos, nacionalidades e classes sociais, causando complicações agudas e crônicas, inabilidades, elevadas taxas de hospitalizações e mortes e significativos agravos econômicos e sociais ${ }^{1}$. $O$ contingente estimado de 11,9 milhões de casos pode atingir 19,2 milhões dos brasileiros em $2035^{2}$.

'Enfermeiro. Residente. Residência em Enfermagem Obstétrica do Hospital Universitário da Grande Dourados. Mato Grosso do Sul, Brasil. E-mail: rdomingos.dom@gmail.com

"Enfermeiro. Doutor. Professor Adjunto. Universidade Federal de Mato Grosso do Sul. Brasil. E-mail: marcos_junior@ufms.br

I'Enfermeiro. Prefeitura Municipal de Dourados. Mato Grosso do Sul, Brasil. E-mail: leandro.hisao@gmail.com

IVEnfermeira. Doutora. Professora Adjunta. Universidade Federal de Mato Grosso do Sul. Brasil. E-mail: marisarolan@gmail.com

vEnfermeira. Doutora. Professora Adjunta. Universidade Federal de Mato Grosso do Sul. Brasil. E-mail: mgorettereis@gmail.com

IVEnfermeiro. Doutor. Professor Adjunto. Universidade Federal de Mato Grosso do Sul. Brasil. E-mail: oleci.frota@ufms.br 
Modificações nos hábitos de vida, como dieta e atividade física, terapêutica medicamentosa regular, insulinoterapia e monitorização glicêmica, são fundamentais para a resposta adequada ao tratamento, mas que nem sempre são de fácil entendimento pela população. Além disso, a falta de conhecimento sobre esta doença agregada à deficiência de estrutura do sistema público de saúde para a atenção integral e multidisciplinar voltada a estes pacientes, contribuem para o aumento dos índices epidemiológicos apresentados ${ }^{3}$.

Visando maior aproximação à fisiologia humana, controle glicêmico aceitável e minimização de episódios hipoglicêmicos, métodos integrados cada vez mais sofisticados de monitoramento dos níveis de glicose e de entrega de insulina estão em fase de projeção e testagem ${ }^{4}$. Há evidências de que a utilização de tecnologias inovadoras pode melhorar o manejo do DM. Para tanto, concentraram-se na aplicação da ciência e tecnologia para permitir a criação de novas e rentáveis ferramentas para ajudar as pessoas com $\mathrm{DM}^{5}$. A exploração das tecnologias da informação e da comunicação (TIC) podem ajudar a reformular o atual sistema de prestação de cuidados de saúde, com deslocamento da ênfase da doença para o bem-estar. Além disso, a utilização dessas novas abordagens oferece algumas vantagens: redução de iatrogenias, geração de dados para investigação da doença e o aumento da capacidade de melhoria contínua 6 .

Entretanto, apesar dos significativos avanços em tecnologias terapêuticas, farmacológicas e de monitoramento ocorridos no século passado e início deste milênio, ainda há grandes desafios para a manutenção de índices glicêmicos aceitáveis, o controle do DM e do estilo de vida saudável. Atualmente, a influência dessas terapêuticas na vida de diabéticos é pouco explorada, com necessidade de estudos de investigação robustos. Assim, este estudo objetivou revisar artigos de pesquisa visando a análise do impacto de tecnologias inovadoras na vida de diabéticos adultos.

\section{METODOLOGIA}

Trata-se de uma revisão integrativa da literatura. Este tipo de estudo consiste em um instrumento da Prática Baseada em Evidências (PBE) que permite a compreensão holística acerca do fenômeno estudado, haja vista que admite estudos experimentais e não experimentais a respeito de uma particular área de estudo. Possibilita a associação entre o conhecimento teórico e empírico, bem como a inclusão de ampla gama de finalidades, como a definição das ideias, revisão de teorias e evidências e a análise de problemas no conjunto de regras de como se deve proceder a fim de produzir conhecimento científico de uma temática em particular ${ }^{7}$.

Para a elaboração desta revisão foram seguidas seis etapas distintas e sequenciais preconizadas na literatura ${ }^{8}$, a saber: 1) identificação do tema e questão de pesquisa; 2) estabelecimento de critérios de inclusão e exclusão dos estudos, com busca na literatura; 3) definição das informações a serem extraídas dos estudos selecionados; 4) avaliação dos estudos incluídos; 5) interpretação dos resultados e; 6) apresentação da revisão. Como ponto de partida, formulouse a seguinte questão norteadora: Qual o impacto proporcionado pelas tecnologias inovadoras na qualidade de vida (QV) de diabéticos adultos?

Para responder esta questão, foi realizada pesquisa eletrônica nas bases de dados Medical Literature Analysis and Retrieval System Online (MEDLINE), Cummulative Index to Nursing nd Allied Health Literature (CINAHL), Web of Science, Springer Link, Literatura Latino Americana e do Caribe em Ciências da Saúde (LILACS) e nas bibliotecas virtuais National Center for Biotechnology Information (NCBI/PubMed) e Scientific Electronic Library Online (SciELO). A busca foi executada no mês de novembro de 2016, com cruzamento dos seguintes Descritores em Ciências da Saúde (DeCS) no formulário básico dos bancos de dados: Diabetes mellitus x Quality of life x Innovations, Therapy x Diabetes mellitus x Quality of life, Diabetes mellitus x Quality of life $\mathrm{x}$ Technolog. O processo de seleção dos estudos desta revisão é ilustrado na Figura 1.

Foram adotados como critérios de inclusão - tratar de estudos cujos delineamentos foram ensaios clínicos randomizados (ECR) bem delineados (nível 2 de evidência) ${ }^{9}$, publicados na íntegra, no período de 2012 a 2016 , nos idiomas Português, Inglês ou Espanhol e que apresentassem a influência das novas tecnologias na vida de diabéticos adultos. Optou-se por ECR em razão do melhor nível de evidência e sua disponibilidade na literatura. Foram excluídos registros duplicados em mais de uma base de dados, quando foi considerado apenas uma vez, realizados com crianças, adolescentes ou animais, que abordassem apenas complicações clínicas do DM e investigações cuja intervenção principal fosse a educação em saúde, haja vista o intuito de analisar apenas tecnologias inovadoras.

A fim de tornar didática a análise dos dados, foi utilizado um quadro sinóptico composto pelas seguintes variáveis: título do artigo, ano e país de publicação, delineamento do estudo, população e variáveis estudadas, terapêutica em estudo e seus resultados. 


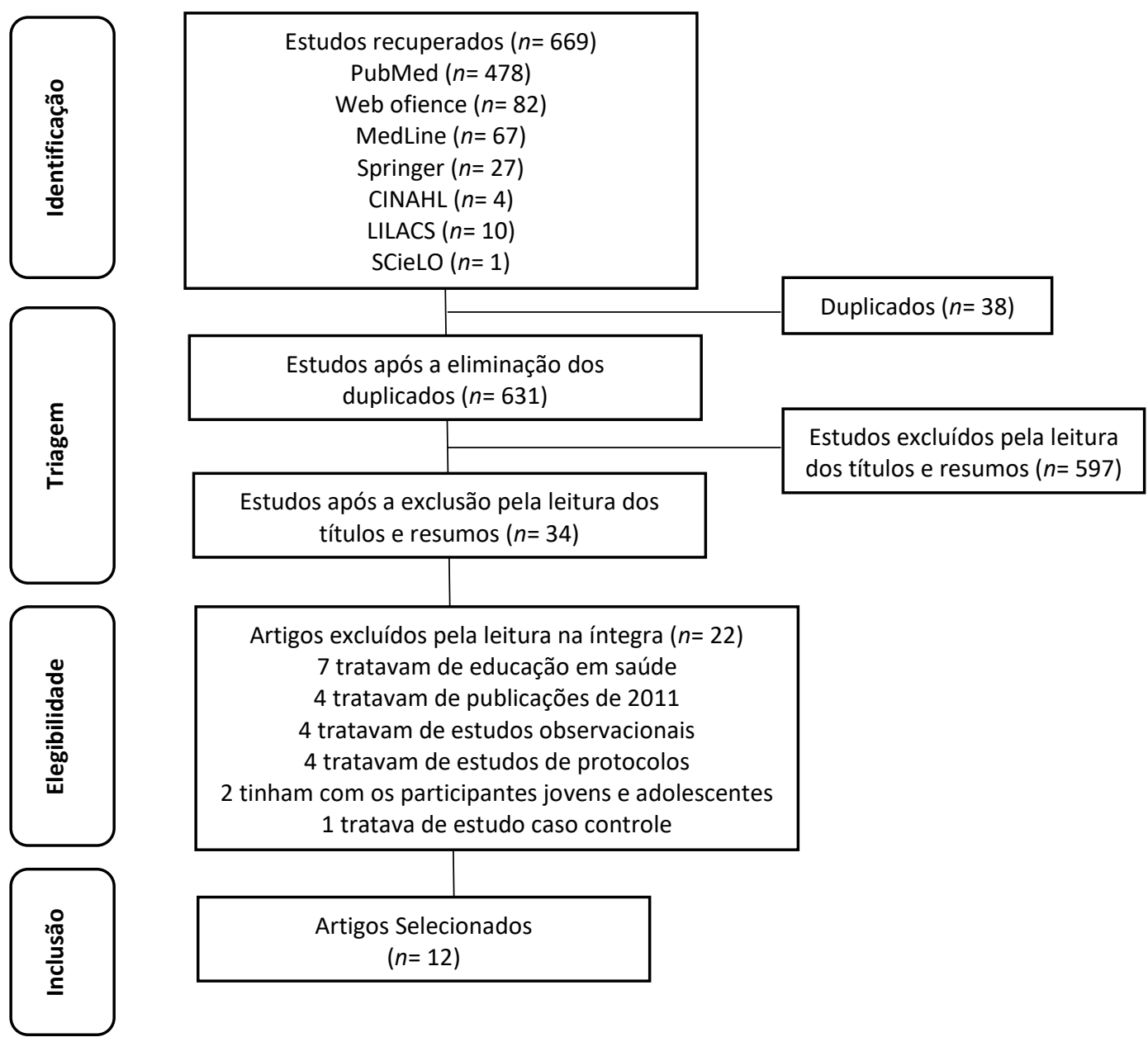

FIGURA 1: Diagrama de busca e seleção dos estudos disponíveis nas bases de dados, elaborado a partir das recomendações PRISMA. Campo Grande, MS, 2016.

\section{RESULTADOS E DISCUSSÃO}

As características dos estudos revisados ${ }^{10-21}$ são apresentadas nas Figura 2 e 3 . Destaca-se que estes foram publicados entre 2013 a 2016, com predominância de produão dos Estados Unidos da América (5/12). Os estudos analisados exploraram 11 diferentes tecnologias inovadoras para o tratamento, vigilância e controle do DM em adultos, que incluíram desde a utilização de aplicativos para smartphones, farmacoterapias, dispositivos de monitorização dos níveis glicêmicos e intervenções. Quase todas as tecnologias (10/11) apresentam consequências benéficas à vida e/ou manejo dos diabéticos. Das tecnologias que revelaram benefícios, apenas o dispositivo de monitorização contínua de glicose, com ou sem função de alarme, Health Coaching, Dapagliflozin e Coolsense não demonstraram aspectos desfavoráveis à vida e manejo, segundo os estudos revisados.

O Aplicativo para smartphone Glucose Buddy é uma ferramenta que auxilia o paciente com DM a assumir um papel ativo no gerenciamento de seus cuidados $^{10}$. Em avaliação geral, ao incluir 10 monitores de glicose baseados em smartphones, o Glucose Buddy foi classificado como fácil de usar, gratuito e notado por seus distintos recursos de lembrete ${ }^{22}$. Os logs de dados do Glucose Buddy fornecem uma maneira eficiente, individualizada e visual para monitorar e controlar o exercício físico, o uso de medicamentos, os níveis séricos de lipídios, a pressão arterial, a ingestão nutricional e a glicemia. 


\begin{tabular}{|c|c|c|}
\hline Terapêutica/ Tecnologia & Autores, revista, ano, país & Resultados encontrados \\
\hline Glucose Buddy & $\begin{array}{l}\text { Kirwan et al. Journal of } \\
\text { Medical Internet Research, } \\
\text { 2013. Austrália }{ }^{10}\end{array}$ & $\begin{array}{l}\text { - Diminui os níveis de HbA1c } \\
\text { - Melhora o controle glicêmico } \\
\text { - Abandono do acompanhamento do controle glicêmico } \\
\text { diário }\end{array}$ \\
\hline $\begin{array}{l}\text { Serviço de } \\
\text { aconselhamento via } \\
\text { telefonia móvel }\end{array}$ & $\begin{array}{l}\text { Mons et al. Plos one, } 2013 . \\
\text { Alemanha }\end{array}$ & $\begin{array}{l}\text { - Diminui os níveis de HbA1c e sintomas de depressão } \\
\text { - Diminui pressão arterial sistólica } \\
\text { - Melhora a QV física } \\
\text { - Mantém os níveis de QV mental do indivíduo } \\
\text { - Redução dos benefícios alcançados quando diabético se } \\
\text { desvincula do serviço de aconselhamento }\end{array}$ \\
\hline Diabetes Integrative & Rossi et al. Diabetes & - Diminui os níveis de HbAc1 e doses de insulina basal \\
\hline Diary: DID & $\begin{array}{l}\text { Technology \& Therapeutics, } \\
\text { 2013. USA }\end{array}$ & $\begin{array}{l}\text { - Melhora o controle metabólico } \\
\text { - Melhora a percepção da frequência de episódios } \\
\text { hiperglicêmicos } \\
\text { - Melhora as relações sociais } \\
\text { - Aumento dos episódios hipoglicêmicos }\end{array}$ \\
\hline CoolSense & $\begin{array}{l}\text { Wainstein et al. Diabetes } \\
\text { Technology \& Therapeutics, } \\
\text { 2013. Israel }\end{array}$ & $\begin{array}{l}\text { - Reduz a sensação de na região de aplicação (dedos) ou } \\
\text { locais de aplicação de insulina subcutânea } \\
\text { - Não foram relatadas alterações negativas na QV e no } \\
\text { manejo }\end{array}$ \\
\hline Dapagliflozin & $\begin{array}{l}\text { Grandy et al. Int J Clin Pract, } \\
\text { 2014. USA }\end{array}$ & $\begin{array}{l}\text { - Mantém níveis elevados do estado de saúde e QV } \\
\text { - Controla a glicemia } \\
\text { - Diminui o peso corporal para nível desejado } \\
\text { - Dificuldade no autocuidado (se lavar e/ou vestir) } \\
\text { - Diminuição da mobilidade } \\
\text { - Alguns participantes referiram dor, desconforto, ansiedade } \\
\text { e sentimento de depressão }\end{array}$ \\
\hline
\end{tabular}

LEGENDA: Diabetes Mellitus: DM; Hemoglobina glicada: HbA1c; Qualidade de vida: QV; United States of América: USA

FIGURA 2: Distribuição dos artigos que abordaram tecnologias inovadoras na vida de diabéticos adultos publicados nos períodos de 2013 a 2014. Brasil, 2018.

Além disso, os logs podem ser totalmente personalizados de acordo com a preferência do usuário e incluem opções para permitir que o indivíduo visualize as médias de glicose durante diferentes horas do dia e forneça uma estimativa de $\mathrm{HbA1c}$. Os logs também permitem que o paciente sobreponha o uso de medicação e exercício sobre os gráficos de glicose que proporciona ao usuário, visualizar como isso afeta sua glicemia. $O$ aplicativo fornece notificações de lembrete caso o usuário não tenha concluído uma tarefa selecionada ${ }^{23}$. Isso explica o seu impacto na diminuição dos níveis de $\mathrm{HbA1c}$ e melhora no controle glicêmico, atenuando a progressão do DM e o surgimento/agravamento de comorbidades.

Esta tecnologia, entretanto, apresentou como fator desfavorável o abandono do controle glicêmico após o paciente utilizá-la por um tempo e cessar o uso ${ }^{10}$. A não adesão ao tratamento pode afetar significativamente a saúde do paciente de forma adversa, acarretando impacto negativo em sua relação com o cuidador, além de aumentar a busca e o consumo de recursos em saúde ${ }^{24}$. Pesquisas mostram que, para incentivar a aceitação e adoção ao longo prazo, as aplicações de smartphones devem ser precisas e amigas do usuário ${ }^{10}$. Isso é especialmente relevante quando se investiga a eficácia das aplicações de smartphones para melhorar o autocuidado em pacientes com DM1.

O Serviço de Aconselhamento Via Telefonia Móvel ofereceu sessões mensais de aconselhamento telefônico conduzidas por enfermeiros. Todas as orientações foram realizadas segundo o manual especificamente construído para este fim e as sessões conduzidas por abordagem proposta por questionário padronizado desenvolvido pelos autores. 0 questionário incluiu perguntas sobre a condição física e mental do paciente, aderência à terapêutica medicamentosa, sintomas e na vida dos diabéticos ${ }^{11}$.

Com esta abordagem, o serviço melhorou os aspectos físico e mental dos participantes, diminuiu efeitos da depressão, níveis de hipertensão arterial sistólica e de HbA1c. Quando há uma intervenção educativa eficaz, na qual o conteúdo abordado consegue ser assimilado pelos participantes, o nível de conhecimento passa a ser maior, como visto no presente estudo, o que permite, posteriormente, um melhor controle da doença por parte dos pacientes. Diversas publicações, em diferentes países, têm reportado a importância de programas educativos para promover maior adesão ao tratamento, resultando em melhor controle do diabetes ${ }^{25}$. 


\begin{tabular}{|c|c|c|}
\hline $\begin{array}{c}\text { Terapêutica/ } \\
\text { tecnologia }\end{array}$ & Autores, revista, ano, país & Resultados encontrados \\
\hline DRMS & $\begin{array}{l}\text { Katalenich et al. Clinical Therapeutics, } \\
\text { 2015. USA }\end{array}$ & $\begin{array}{l}\text { - Ausência de eventos adversos } \\
\text { - Melhora a QV social e profissional } \\
\text { - Potencializa a adesão à terapêutica } \\
\text { - Dificuldades técnicas durante as chamadas e } \\
\text { operacional para os tipos e doses de insulina }\end{array}$ \\
\hline DBEES & $\begin{array}{l}\text { Drion et al. Journal of Diabetes Science } \\
\text { and Technology, 2015. Holanda }\end{array}$ & - Sem mudanças nos níveis de HbA1c e QV \\
\hline Health Coaching & $\begin{array}{l}\text { Wayne et al. Journal of Medical } \\
\text { Internet Research, 2015. Canadá }\end{array}$ & $\begin{array}{l}\text { - Reduz o peso corporal e a circunferência abdominal } \\
\text { - Melhora a satisfação com a vida e o humor } \\
\text { - Reduz Hb1Ac e sintomas da depressão e ansiedade } \\
\text { - Melhora o bem-estar psicológico } \\
\text { - Aumenta os cuidados habituais e o prestígio da } \\
\text { importância da prática de exercícios físicos }\end{array}$ \\
\hline $\begin{array}{l}\text { Dispositivo de } \\
\text { monitorização } \\
\text { contínua de glicose }\end{array}$ & $\begin{array}{l}\text { New et al. Diabetic Medicine, } 2015 \\
\text { Reino Unido }\end{array}$ & $\begin{array}{l}\text { - Reduz níveis de HbA1c, episódios de hipoglicemia e } \\
\text { frequência das medições capilares }\end{array}$ \\
\hline Dapagliflozin & $\begin{array}{l}\text { Grandy et al. Diabetes, Obesity and } \\
\text { Metabolism, 2016. USA }\end{array}$ & $\begin{array}{l}\text { - Elimina o excesso de gordura corporal } \\
\text { - Melhora o estado de saúde geral e a QV } \\
\text { - Acarreta satisfação com o tratamento }\end{array}$ \\
\hline $\begin{array}{l}\text { Automates Bolus } \\
\text { Calculator }\end{array}$ & $\begin{array}{l}\text { Hommel et al. Diabetic Medicine, } 2016 . \\
\text { Reino Unido }\end{array}$ & $\begin{array}{l}\text { - Melhora o controle metabólico e níveis de HbA1c } \\
\text { - Oferece baixo risco de efeitos colaterais } \\
\text { - Proporciona satisfação com o tratamento }\end{array}$ \\
\hline Empagliflozina & Chirila et al. Qual Life Res, 2016 USA & $\begin{array}{l}\text { - Reduz e sustenta os níveis de HbA1c } \\
\text { - Ocorrência de efeitos adversos, principalmente } \\
\text { hiperglicemia, hipertensão e infecção urinária } \\
\text { - Episódios de hipoglicemia com o uso da medicação }\end{array}$ \\
\hline
\end{tabular}

LEGENDA: Diabetes Mellitus: DM; Hemoglobina glicada: HbA1c; Qualidade de vida: QV; United States of América: USA

FIGURA 3: Distribuição dos artigos que abordaram tecnologias inovadoras na vida de diabéticos adultos publicados nos períodos de 2015 a 2016. Brasil, 2018.

Todavia, quando o paciente deixou de utilizar o serviço de aconselhamento, ocorreu diminuição dos efeitos benéficos previamente alcançados, isto é, as intervenções não foram capazes de promover mudanças definitivas de comportamentos $^{11}$. Estas mudanças só serão permanentes se acompanhadas de políticas públicas de saúde de abrangência populacional e de acompanhamento efetivo da atenção primária à saúde.

O Diabetes Interactive Diary (DID) é um software para celulares que funciona como uma calculadora de bolus de insulina. Ela fornece suporte aos pacientes na gestão dos níveis glicêmicos por meio de um atlas de alimentos e na gravação dos valores obtidos pela automonitorização da glicemia. Com base nos dados armazenados, o aplicativo calcula automaticamente a dose de insulina mais apropriada para ser injetada após cada refeição. Todos os dados gravados são enviados ao médico via SMS. Qualquer nova prescrição terapêutica e comportamental pode ser enviada do computador da clínica para o celular do paciente ${ }^{12}$.

A tecnologia DID apresentou como vantagens a melhoria no controle metabólico e na percepção da frequência de episódios hiperglicêmicos, a diminuição significativa de $\mathrm{HbAc1}$ e das doses diárias de insulina basal e o fortalecimento nas relações sociais ${ }^{12}$. A melhora no controle metabólico é obtida ao mesmo tempo que melhora a QV em termos de percepção de hiperglicemia, medo de hipoglicemia e satisfação com as relações sociais. A melhoria na QV e na satisfação com o tratamento representam um objetivo chave do cuidado de qualquer doença crônica ${ }^{26,27}$. Entretanto, a tecnologia apresentou aspecto desfavorável relacionado ao estilo de vida e controle do DM pela alta frequência de episódios hipoglicêmicos ${ }^{12}$.

O CoolSense é composto por dispositivo de contato que, ao ser aplicado à pele, anestesia o local e reduz a dor à realização da glicemia e da insulinoterapia. Apenas o benefício esperado de redução significativa da sensação de dor foi relatado para esta tecnologia ${ }^{13}$. O uso do dispositivo CoolSense diminuiu significativamente a dor causada por agulhamento e os pacientes também queriam usar o dispositivo para monitoramento diário. Entretanto, não foram observadas diferenças nos níveis de glicemia, uma vez que o dispositivo não altera a glicemia de forma clinicamente significativa ${ }^{13}$. 
A Dapagliflozina é um fármaco, em processo de teste, desenvolvido para o tratamento de DM2. Bloqueia o cotransportador sódio-glicose 2 (SGLT2), proteína responsável pela reabsorção da glicose no rim e leva à eliminação do excesso de glicose na urina e melhora o controle do diabetes. Vários estudos já demonstraram que o SGLT2 pode reduzir o peso corporal em pacientes com DM2, uma vez que a eliminação da glicose pela urina leva à perda de calorias (em torno de 200 a $300 \mathrm{cal} / \mathrm{dia}$ ), resultando em um balanço energético negativo ${ }^{28}$.

A tecnologia esteve associada a importantes inconvenientes classificados de leve a moderados, como as dificuldades no autocuidado (lavar-se/ou vestir-se), dor, desconforto, ansiedade, diminuição da mobilidade e sentimento de depressão. Apesar disso, impactou em níveis elevados no estado geral de saúde autorreferida e estilo de vida, na diminuição da glicemia e do peso corporal, assim como na satisfação com o tratamento ${ }^{14,19}$. Estes dados chamaram atenção, pois mesmo diante de vários aspectos desfavoráveis ao estilo de vida, a terapia com a Dapagliflozina agradou aos participantes.

O Diabetes Remote Monitoring and Management System (DRMS) usa tecnologia (telefones celulares e/ou fixos) para minimizar o uso de recursos humanos. O sistema utiliza tecnologia baseada em regras para automatizar o cuidado manual e monitorar planos de cuidados, criando ações e intervenções personalizadas com base em parâmetros definidos pelo médico e no protocolo médico específico. O sistema foi projetado para entrar em contato com o paciente diariamente, por meio de mensagens de texto ou voz automatizadas. A partir dessas mensagens, os usuários poderiam informar seus níveis de glicose no sangue ou responder mais tarde. O sistema também os lembra de monitorar a glicose e ingerir os medicamentos, além de alertar os profissionais quando surgem problemas ${ }^{15}$.

O DRMS impactou positivamente no estilo de vida e na eficácia do tratamento e controle da DM, pois foi evidenciada a ausência de eventos adversos secundários, com melhoria no estilo de vida social e profissional e maior adesão ao tratamento medicamentoso. Compreende-se que o empoderamento do paciente portador de diabetes para o autocuidado melhora consideravelmente a adesão ao tratamento e, consequentemente, seu quadro clínico ${ }^{29}$. Apesar de boa satisfação da clientela, a tecnologia apresentou obstáculos operacionais, como o contratempo de operação adequada para todos os tipos e doses de insulinas e dificuldades técnicas durante as chamadas automatizadas.

Nesse sentido, um estudo apontou que esses problemas representam um desafio para os usuários e pode fazer com que eles percam o interesse em usar seus dispositivos móveis ${ }^{30}$. Outro estudo registrou que os recursos adicionados ao design do aplicativo podem comprometer a capacidade do usuário de alcançar seus objetivos, pois aumenta a complexidade e diminui a usabilidade ${ }^{31}$. Portanto, observa-se a necessidade de aprimoramentos nesta tecnologia, com vistas à simplicidade, eficiência e, logo, maior adesão.

O sistema Diabetes Under Control (DBEES) é um aplicativo para smartphones que fornece aos pacientes dados relacionados ao autocuidado com o DM. Com o aplicativo, é possível armazenar os valores de glicemia e calcular a taxa de carboidrato ingerida na alimentação. O sistema contém a função de alarme, que avisa ao paciente o horário da medicação. Estudos verificaram que programas de educação em diabetes, que incluíam terapia nutricional e planos de cuidado individualizados tiveram associação com a diminuição do $\mathrm{HbA1c}$ em pessoas com $\mathrm{DM}^{32}$. Contudo, o DBEES não promoveu mudanças nos níveis de $\mathrm{HbA} 1 \mathrm{c}$ e, consequentemente, não foram encontradas alterações na vida dos diabéticos ${ }^{16}$.

O Serviço Health Coaching é definido como treinamento em saúde, cujo especialista em mudança de comportamento oferece serviços de aconselhamento aos diabéticos. Os treinadores apresentam experiência em manejo de doenças crônicas e teoria baseada em evidências adaptada para o estado da doença. Com a assistência do treinador em saúde, os clientes determinam metas relacionadas à saúde e acompanham o progresso diário. Esta tecnologia apresentou inúmeros benefícios relacionados ao estilo de vida, tratamento, prevenção de complicações, vigilância e controle do DM e não foi observado qualquer aspecto desfavorável ${ }^{17}$.

Essas intervenções educativas destinadas a facilitar o desenvolvimento de habilidades específicas para o enfrentamento do diabetes podem melhorar a QV dos pacientes com essa doença ${ }^{33}$.

O Dispositivo de Monitorização Invasiva Contínua de Glicose (MGC), com ou sem função de alarme, é uma tecnologia emergente que fornece uma medida contínua de níveis de glicose intersticial. Além de fornecer um padrão mais completo de excursões de glicose, o MGC utiliza alarmes em tempo real para valores correspondentes à hipoglicemia e hiperglicemia, bem como alarmes para as rápidas mudanças glicêmicas. Estes dispositivos utilizam sensores subcutâneos eletroquímicos baseados em glicose-oxidase para detectar níveis de glicose no fluido intersticial. Uma corrente elétrica é gerada à medida que a glicose é oxidada pelo sensor e a corrente elétrica é então transmitida para o receptor ou monitor. Os dados são filtrados para remover o ruído do sensor e em seguida é fornecido o valor da dosagem de glicose sanguínea ${ }^{18}$. 
Redução dos episódios de hipoglicemia e da frequência de medições capilares, menor tempo em estado de hipoglicemia e menor variação nos níveis glicêmicos foram os aspectos positivos desse dispositivo. Entretanto, embora esta tecnologia tenha resultado positivo na redução de episódios de hipoglicemia, não houve alterações significativas na concentração de $\mathrm{HbA1c}$ a partir da linha de base, o que indica que a hipoglicemia reduzida não foi causada pela piora do controle glicêmico ${ }^{18}$.

O Automates Bolus Calculator é uma tecnologia de aconselhamento de insulinoterapia que realiza o cálculo preciso da dose de insulina rápida em apenas três passos, pela aferição da glicemia, introdução da quantidade de hidratos de carbono e pela obtenção instantânea do bolus de insulina sem cálculos manuais. O dispositivo tem função de memória e diferentes possibilidades de exibição gráfica para dados armazenados, melhora significativamente o controle metabólico e os níveis de $\mathrm{HbA1c}$, tem baixo risco para efeitos colaterais e os diabéticos apresentam satisfação com o tratamento terapêutico. Não foram relatados aspectos desfavoráveis ao estilo de vida ${ }^{20}$.

A Empagliflozina também é uma droga antidiabética oral que inibe seletivamente o cotransportador SGLT-2 e aumenta a excreção urinária de glicose bloqueando reabsorção de glicose pelo rim $^{21,34}$. A redução sustentada dos níveis de $\mathrm{HbA} 1 \mathrm{c}$ foi o único impacto positivo imediato proporcionado aos diabéticos que fizeram seu uso. Essa abordagem, entretanto, teve como inconvenientes uma frequência maior de efeitos adversos como a hiperglicemia, hipertensão e a infecção urinária, com episódios de hipoglicemia no decorrer do uso das medicações ${ }^{21}$. Um estudo registrou que a frequência de hipoglicemia observada no uso de Empagliflozina variou de acordo com a terapia antidiabética de fundo ${ }^{34}$. Os doentes com DM2 estão em maior risco de sofrer infecções do trato urinário e infecções genitais. Adicionalmente, um ligeiro aumento no risco de infecções do trato urinário tem sido descrito em doentes tratados com Empagliflozina, sobretudo no sexo feminino. Contudo, tratam-se de infecções do trato urinário baixo, que respondem bem à terapêutica preconizada ${ }^{34}$.

As limitações deste estudo residem no número reduzido de ECR sobre cada uma das tecnologias inovadoras, indicando uma lacuna nas evidências sobre o uso das referidas abordagens, bem como na inclusão de artigos apenas nos idiomas Português, Inglês e Espanhol, o que pode ter excluído estudos importantes de outros idiomas.

\section{CONCLUSÃO}

Considerando os artigos revisados, as principais tecnologias inovadoras empregadas no tratamento, vigilância e controle do DM desempenham um papel crucial no desenvolvimento de estratégias para melhorar os resultados da saúde de diabéticos adultos. Foi observada uma influência positiva dessas tecnologias no manejo e na vida dos clientes, uma vez que praticamente todas elas reduziram significativamente os níveis de $\mathrm{Hb} 1 \mathrm{Ac}$, bem como proporcionaram um melhor controle metabólico e glicêmico. No entanto, várias questões abertas e desafios técnicos são identificados como fatores-chave para adotar plenamente esses sistemas na prática clínica.

Apesar dos grandes avanços alcançados na terapia do DM, ainda são necessários estudos com desenhos mais robustos com vistas a alcançar o método de controle glicêmico e de administração de insulina mais adequado a cada pessoa e que favoreça a QV.

\section{REFERÊNCIAS}

1. Crause DH, Soares AR, Amorim RF, Gradella DBT, Souza MAA. Diabetes mellitus in users of the Brazilian Unified Health System in São Mateus, Espírito Santo State, Brazil. Scientia Vitae. 2016 [cited 2018 Sep 17]; 11(3):79-87. Available from: http://www.revistaifspsr.com/marcojan16.pdf

2. Iser BPM, Stopa SR, Chueiri PS, Szwarcwald CL, Malta DC, Monteiro HOC, et al. Self-reported diabetes prevalence in Brazil: results from National Health Survey 2013. Epidemiol. serv. saúde. 2015 [cited 2018 Sep 17]; 24(2):305-14. DOI: http://dx.doi.org/10.5123/S1679-49742015000200013

3. Sousa EL, Martins MM, Costa MS, Moreira MRC, Silva AO. Quality of life and factors associated with the health of elderly diabetics Rev. enferm. UERJ. 2016 [cited 2019 Aug 05]; 24(5):e8456. DOI: http://dx.doi.org/10.12957/reuerj.2016.8456

4. Riemsma R, Ramos IC, Birnie R, Büyükkaramikli N, Armstrong N, Ryder S, et al. Integrated sensor-augmented pump therapy systems [the MiniMed ${ }^{\circledR}$ Paradigm $^{\mathrm{TM}}$ Veo system and the Vibe ${ }^{\mathrm{TM}}$ and G4 ${ }^{\oplus}$ PLATINUM CGM (continuous glucose monitoring) system] for managing blood glucose levels in type 1 diabetes: a systematic review and economic evaluation. Health technol. assess. 2016 [cited 2018 Sep 17]; 20(17):1-251. DOI: https://doi.org/10.3310/hta20170

5. Zarkogianni K, Litsa E, Mitsis K, Wu PY, Kaddi CD, Cheng CW, et al. A review of emerging technologies for the management of diabetes mellitus. IEEE trans. biomed. eng. 2015 [cited 2018 Sep 17]; 62(12):2735-49. DOI: https://doi.org/10.1109/TBME.2015.2470521

6. Riazi H, Larijani B, Langarizadeh M, Shahmoradi L. Managing diabetes mellitus using information technology: a systematic review. J. diabetes metab. disord. 2015 [cited 2018 Sep 17]; 14(49): 1-9. DOI: https://doi.org/10.1186/s40200-015-0174-x

7. Frota OP, Borges NMA. Hemodialysis treatment-related chronic complications in hypertensive people: integrative review. Rev. pesqui. cuid. fundam. 2013 [cited 2018 Sep 17]; 2(5):3828-36. DOI: http://doi.org/10.9789/2175-5361.2013v5n2p3828 
8. Mendes KDS, Silveira RCCP, Galvão CM. Integrative literature review: a research method to incorporate evidence in health care and nursing. Texto \& contexto enferm. 2008 [cited 2018 Sep 17]; 17(4):758-64. DOI: http://dx.doi.org/10.1590/S010407072008000400018

9. Melnyk BM, Fineout-Overholt E. Evidence based practice in nursing \& healthcare: a guide to best practice. Philadelphia (USA): Wolters Kluwer/Lippincott Williams \& Wilkins. 2011 [cited 2018 Sep 17]. Available from: http://file.zums.ac.ir/ebook/208Evidence-Based\%20Practice\%20in\%20Nursing\%20\&\%20Healthcare\%20\%20A\%20Guide\%20to\%20Best\%20Practice,\%20Second\%20Edition-Be.pdf

10. Kirwan M, Vandelanotte C, Fenning A, Duncan MJ. Diabetes self-management smartphone application for adults with type 1 diabetes: randomized controlled trial. J. med. internet. res. 2013 [cited 2018 Sep 17]; 15(11):e235. DOI: https://doi.org/10.2196/jmir.2588

11. Mons U, Raum E, Krämer HU, Rüter G, Rothenbacher D, Rosemann T, et al. Effectiveness of a supportive telephone counseling intervention in type 2 diabetes patients: randomized controlled study. Plos one. 2013 [cited 2018 Sep 17]; 8(10):e77954. DOI: https://doi.org/10.1371/journal.pone.0077954

12. Rossi MC, Nicolucci A, Lucisano G, Pellegrini F, Di Bartolo P, Miselli V, et al. Impact of the 'Diabetes Interactive Diary' telemedicine system on metabolic control, risk of hypoglycemia, and quality of life: a randomized clinical trial in type 1 diabetes. Diabetes technol. ther. 2013 [cited 2018 Sep 17]; 15(8):670-9. DOI: https://doi.org/10.1089/dia.2013.0021

13. Wainstein J, Chimin G, Landau Z, Boaz M, Jakubowicz D, Goddard G, et al. The use of a CoolSense device to lower pain sensation during finger pricking while measuring blood glucose in diabetes patients - a randomized placebo. Diabetes technol. ther. 2013 [cited 2018 Sep 17]; 15(8):688-94. DOI: http://online.liebertpub.com/doi/abs/10.1089/dia.2012.0278

14. Grandy S, Langkilde AM, Sugg JE, Parikh S, Sjöström CD. Health-related quality of life (E.Q.-5 D.) among type 2 diabetes mellitus patients treated with dapagliflozin over 2 years. Int. j. clin. pract. 2014 [cited 2018 Sep 17]; 68(4):486-94. DOI: https://doi.org/10.1111/ijcp.12341

15. Katalenich B, Shi L, Liu S, Shao H, McDuffie R, Carpio G, et al. Evaluation of a remote monitoring system for diabetes control. Clin. ther. 2015 [cited 2018 Sep 17]; 37(6):1216-25. DOI: https://doi.org/10.1016/j.clinthera.2015.03.022

16. Drion I, Pameijer LR, van Dijk PR, Groenier KH, Kleefstra N, Bilo HJ. The effects of a mobile phone application on quality of life in patients with type 1 diabetes mellitus: a randomized controlled trial. J. diabetes sci. technol. 2015 [cited 2018 Sep 17]; 9(5):108691. DOI: https://doi.org/10.1177/1932296815585871

17. Wayne N, Perez DF, Kaplan DM, Ritvo P. Health coaching reduces hba1c in type 2 diabetic patients from a lower-socioeconomic status community: a randomized controlled trial. J. med. internet res. [Internet]. 2015 [cited 2018 Sep 17]; 17(10):e224, 1-19. DOI: https://doi.org/10.2196/jmir.4871

18. New JP, Ajjan R, Pfeiffer AF, Freckmann G. Continuous glucose monitoring in people with diabetes: the randomized controlled glucose level awareness in diabetes study (G.L.A.D.I.S.). Diabet. med. 2015 [cited 2018 Sep 17]; 32(5):609-17. DOI: https://doi.org/10.1111/dme.12713

19. Grandy S, Sternhufvud C, Ryden A, Sugg J, Rohwedder K. Patient-reported outcomes among patients with type 2 diabetes mellitus treated with dapagliflozin in a triple-therapy regimen for 52 weeks. Diabetes obes. metab. 2016 [cited 2018 Sep 17]; 18(3):3069. DOI: https://doi.org/10.1111/ijcp.12341

20. Hommel E, Schmidt S, Vistisen D, Neergaard K, Gribhild M, Almdal T, et al. Effects of advanced carbohydrate counting guided by an automated bolus calculator in type 1 diabetes mellitus (Steno A.B.C.): a 12-month, randomized clinical trial. Diabet. med. 2016 [cited 2018 Sep 17]; 34(5):708-15. DOI: https://doi.org/10.1111/dme.13275

21. Chirila C, Zheng $Q$, Davenport E, Kaschinski D, Pfarr E, Hach T, et al. Treatment satisfaction in type 2 diabetes patients taking empagliflozin compared with patients taking glimepiride. Qual. life. res. 2016 [cited 2018 Sep 17]; 25(5):1199-207. DOI: https://doi.org/10.1007/s11136-015-1140-2

22. Tran J, Tran R, White J. Smartphone-based glucose monitors and applications in the management of diabetes: an overview of 10 salient "apps" and a novel smartphone connected blood glucose monitor. Clin. diabet. 2012 [cited 2018 Sep 17]; 30(4):173-8. DOI: https://doi.org/10.2337/diaclin.30.4.173

23. Randall A. Integrating technology to support and maintain glycemic control in people with diabetes [dissertation]. Anchorage, Alaska, USA: Faculty of the University of Alaska Anchorage. 2016 [cited 2018 Sep 17]. Available from: https://scholarworks.alaska.edu/handle/11122/6746

24. Dayer L, Heldenbrand S, Anderson P, Gubbins PO, Martin BC. Smartphone medication adherence apps: potential benefits to patients and providers. J. am. pharm. assoc. 2013 [cited 2018 Sep 17]; 53(2):172-81. DOI: http://doi.org/10.1331/JAPhA.2013.12202

25. Barbosa L, Borges PCP, Lemos SS, Cesarino CB. Evaluation of group educational intervention for diabetics receiving care at teaching clinic. Rev. enferm. UERJ. 2016 [cited 2018 Aug 05]; 24(2):e4968. DOI: http://dx.doi.org/10.12957/reuerj.2016.4968

26. Nicolucci A, Cucinotta D, Squatrito S, Lapolla A, Musacchio N, Leotta S, et al. Clinical and socio-economic correlates of quality of life and treatment satisfaction in patients with type 2 diabetes. Nutr. metab. cardiovasc. dis. 2009 [cited 2018 Sep 17]; 19(1):4553. DOI: https://doi.org/10.1016/j.numecd.2007.12.005

27. Saatci E, Tahmiscioglu G, Bozdemir N, Akpinar E, Ozcan S, Kurdak H. The well-being and treatment satisfaction of diabetic patients in primary care. Health qual. life outcomes. 2010 [cited 2018 Sep 17]; 8(67):1-8. DOI: https://doi.org/10.1186/1477-7525-8-67

28. Santos LL, Lim, FJC, Rodrigues CFS, Barbosa FT. Use of SGLT-2 inhibitors in the treatment of type 2 diabetes mellitus. Rer. assoc. med. bras. 2017 [Cited 2019 Aug 06]; 63(7):636-41. DOI: http://dx.doi.org/10.1590/1806-9282.63.07.636 
29. Amaral RT, Barbosa AM, Teixeira CG, Brandão, LGVA, Afonso AM, Bezerra ALQ. Knowledge of diabetics about disease and selfcare. Rev. enferm. UFPE. 2019 [Cited 2019 Aug 05]; 13(1):346-52. DOI: https://doi.org/10.5205/1981-8963-v13i02a239077

30. Shin HSW, Dhahir NF, Mohan JAL, Abdullah NL. Emergency related mobile applications: usability framework and proposed implementation. Int. j. n. comp. arch. app. 2015 [cited 2018 Sep 17]; 5(4):165-74. DOI: http://dx.doi.org/10.17781/P001984

31. Harrison R, Flood D, Duce D. Usability of mobile applications: literature review and rationale for a new usability model. J. interact. sci. 2013 [cited 2018 Sep 17]; 1(1):2-16. DOI: http://dx.doi.org/10.1186/2194-0827-1-1

32. Rossaneis MA, Andrade SM, Gvozd R, Pissinati PSC, Haddad, MCL. Factors associated with glycemic control in people with diabetes mellitus. Ciência \& saúde coletiva. 2019 [cited 2018 Aug 05]; 24(3):997-1005. DOI: http://dx.doi.org/10.1590/141381232018243.02022017

33. Corrêa K, Gouvêa GR, Silva MAV, Possobon RF, Barbosa LFLN, Pereira AC, et al. Quality of life and characteristics of diabetic patients. Ciência \& saúde coletiva. 2017 [cited 2018 Aug 05]; 22(3):921-30. DOI: http://dx.doi.org/10.1590/141381232017223.24452015

34. Calado J, Nunes JS. Empagliflozina: uma nova arma farmacológica na terapêutica do diabetes tipo 2. Rev. port. diab. 2015 [cited 2018 Sep 17]; 3(10):118-26. Available from: http://www2.spd.pt:8080/wp-content/uploads/2013/04/rpdset15.pdf 